\title{
Moi, médecin, 30 ans, en crise
}

\section{Mirjam Tanner}

Dr med., spécialiste en psychiatrie et psychothérapie, Comité de direction de ReMed

Le témoignage présenté ci-dessous décrit de manière exemplaire une situation rencontrée régulièrement par le réseau de soutien ReMed: celle de jeunes femmes médecins en formation, particulièrement compétentes, sensibles et engagées, portées par des idéaux élevés qui, frustrées par leur quotidien, remettent en question leur choix de carrière. L'auteur lance ici un appel à soutenir nos jeunes consœurs afin de ne pas les perdre au cours de leur formation.

«En m'adressant à ReMed, j'ai bien cru que j'allais m’effondrer ou être dévorée vivante par un monstre. Mon cœur s'est emballé, mon t-shirt était trempé de sueur et tout tournait autour de moi. Heureusement, la voix apaisante du conseiller de ReMed au téléphone m'a quelque peu rassurée et je suis parvenue à lui expliquer ma situation. J'ai 32 ans, je termine ma formation d'interniste, j'ai commencé mon dernier stage il y a deux mois, et... je n'en peux tout simplement plus. Tout n'est que torture, angoisse, peur, stress, reproches envers mes heures supplémentaires... plus rien n'a de sens. J'admire les autres qui parviennent à y faire face aussi facilement. J'ai l'impression de ne pas être faite pour ce travail, et je suis très déçue de moi-même.

Pour moi, devenir médecin est un rêve de petite fille. Aider les autres, sauver des vies, particulièrement celles d'enfants dont personne ne s'occupe. Depuis, je suis bien devenue médecin, mais un médecin tellement stressé par le quotidien qu'il ne trouve plus le temps pour s'occuper de ses patients et pour vraiment les écouter. Comme si des milliers de personnes exigeaient toutes en même temps quelque chose de moi, et ce tout de suite. Or je suis consciencieuse, appliquée, et pas multitâche.

\section{Epuisée, désillusionnée, désorientée}

En ce moment, je suis tellement sur les nerfs que j'en deviens impatiente, désagréable et particulièrement distante. Je réalise qu'il m'arrive même de brusquer certains patients. Qu'est-il advenu de mon rêve de petite fille? Une fois, un de mes chefs m'a dit que j'avais des idéaux trop élevés et que je voulais trop bien faire mon travail. Je devrais apprendre à me distancer et à ne pas vouloir tout recontrôler et tout remettre en question, mais avoir confiance en mes décisions. Mais

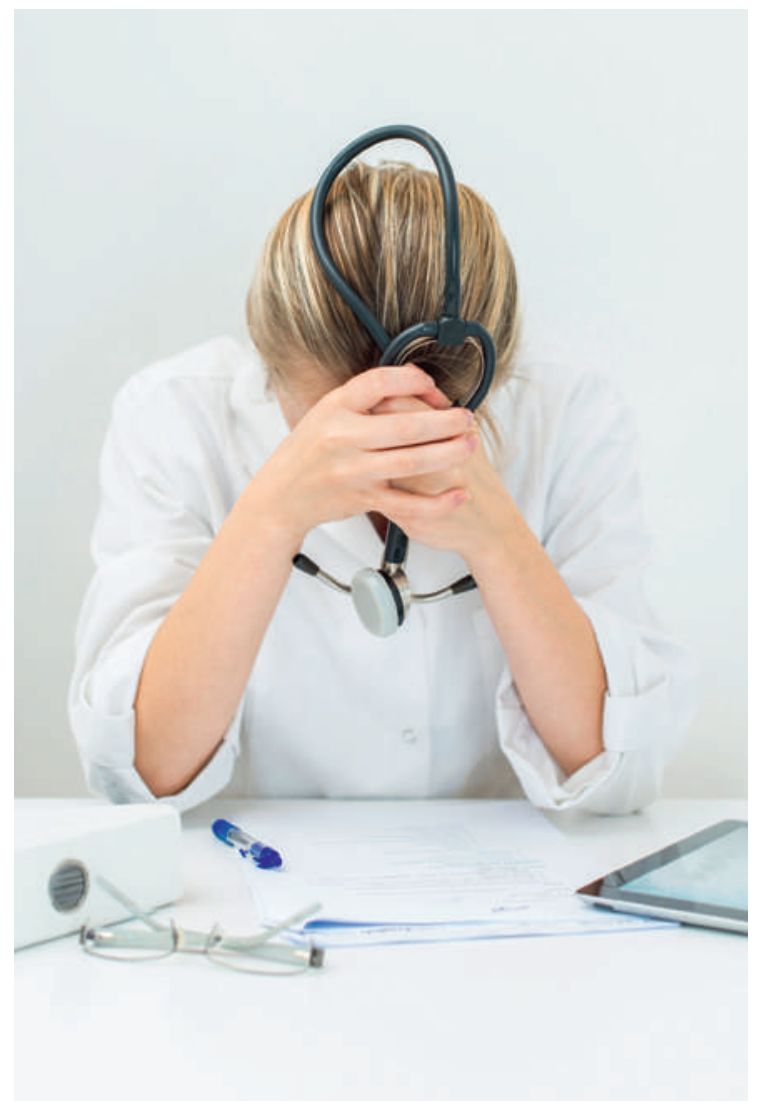

ReMed vous aide à remonter la pente lorsque vous êtes au creux de la vague.

\section{ReMed est là pour vous}

Avez-vous ou quelqu'un de votre entourage a-t-il besoin d'un soutien professionnel? Dans ce cas, adressez-vous à ReMed: le réseau de soutien pour les médecins vous conseille de manière compétente dans le respect du secret médical. ReMed peut également intervenir dans d'autres situations de crise professionnelles ou personnelles. L'équipe de conseillères et conseillers se manifeste dans les 72 heures: www.swissremed.ch, help[at]swiss-remed.ch, tél. 0800073633. 
depuis qu'une de mes patientes est décédée d'une embolie pulmonaire, que la cheffe de clinique n'avait pas non plus diagnostiquée, je lutte chaque jour contre ma peur de louper quelque chose. Chaque matin, au moment de passer la porte de la clinique, je suis submergée par les doutes et j'ai l'impression que tout le monde sait que je suis un mauvais médecin. Même si mon chef ne me l'a jamais dit. Je ne comprends d'ailleurs pas pourquoi il a autant confiance en mes capacités ni surtout pourquoi il m'a proposé de m'engager dans son service. C'est difficile à dire mais j'ai l'impression qu'en faisant cela, il démontre sa propre incompétence.

\section{Solitude, tristesse, peur de ne pas pouvoir fonder une famille}

Lorsque mon interlocuteur de ReMed m'a demandé si j'avais des contacts sociaux, il m'a fallu un moment pour répondre. J'ai soudain pris conscience de la situation et les larmes se sont mises à couler. Ce n'est pas une question anodine. Mon dernier rendez-vous, c'était il y a deux mois avec ma sœur. J'ai bien une amie dans une autre ville, à qui je téléphone de temps en temps. Mais depuis que je travaille à l'hôpital, j'ai abandonné la musique. Avant, je chantais dans un groupe

\section{Je lutte chaque jour contre ma peur de louper quelque chose.}

mais j'ai vite dû abandonner faute de pouvoir m'exercer en raison de mes heures de travail irrégulières. De plus, je n'ai jamais vraiment été satisfaite de mes prestations de chanteuse. Je suis très exigeante envers moimême, non seulement au travail mais également dans tous les autres domaines.

Comme je suis quelqu'un de compliqué, mes relations avec les hommes s'en ressentent également. Je cache une grande tristesse et je nourris de grandes aspirations. J'ai honte de dire que cela fait quatre ans que je suis célibataire, et que chaque année, l'angoisse augmente de ne pas pouvoir réaliser mon rêve d'avoir des enfants. Comment trouver le bon partenaire dans ces conditions? Je me sens impuissante, condamnée à ne pas être aimée ni assez intéressante pour les hommes qui m'intéresseraient. Ma dernière histoire, c'était avec un collègue pour qui j'avais une grande admiration. Nous sommes restés ensemble trois ans. C'est lui qui a soudain mis fin à notre relation en raison de notre vision différente de la vie. Depuis, il est devenu père et s'est marié avec une infirmière, qui a arrêté de travail-

\section{Reprendre confiance}

Même si je pense chaque jour à quitter mon travail et à faire tout autre chose, je sais pertinemment que cela ne me rendrait pas plus heureuse. J'ai demandé au conseiller de ReMed comment faire pour sortir la tête de l'eau. Nous avons reconnu ensemble que, vu de l'extérieur, je faisais du bon travail mais que j'avais l'impression de ne jamais en faire assez. Certes, mon travail est stressant. Mais une grande partie de la pression que je ressens et qui m'angoisse autant, c'est moi-même qui me la mets toute seule. Au bout d'un moment, il n'est plus possible d'y faire face et tout s'effondre. Mais ma situation personnelle peu satisfaisante contribue également à cette fatigue et à ce stress permanent.

Je ne sais pas encore comment je vais m'y prendre pour retrouver ma joie de vivre. Mais au moins, ces deux entretiens avec le conseiller de ReMed m'ont aidée à admettre ouvertement mon désir de rencontrer quelqu'un et de fonder une famille, et à l'accepter comme faisant partie de moi-même. Nous avons discuté de différentes possibilités de soutien dont un tutorat, un coaching ou encore une psychothérapie. Je n'ai pas encore pris ma décision et je ne sais pas non plus si je souhaite entamer cette démarche. Ce contact avec ReMed m'a permis d'y voir un peu plus clair et de gagner en sérénité. Cela me rassure également de savoir que si je décide de chercher de l'aide auprès d'un professionnel, je pourrais sans hésiter m'adresser à nouveau à ReMed et que je n'ai pas à en avoir honte. Cela me sera d'une grande aide de pouvoir me tourner vers une personne expérimentée en qui je peux avoir confiance.»

\section{Crédit photo:}

(c) Dmitri Maruta | Dreamstime.com

\section{Groupes d'intervision: dates 2015}

En 2009, ReMed a lancé des intervisions entre pairs pour répondre à la demande de confrères et depuis cette date, il en organise régulièrement (groupe de 6 à 10 personnes, 2-3 fois/ an). Les participants traitent ensemble leurs questions liées au mentorat, au coaching, au conseil, au traitement / à la thérapie et à d'autres aspects de l'accompagnement collégial (droit, droit des assurances, etc.). Mettez-vous en contact avec nous ou participez à une séance pour vous familiariser avec notre travail. Contact: Peter Birchler, tél. 0443420910 ou peter. birchler[at]hin.ch

Dernières dates en 2015: 12.11. Hôtel Central Plaza, Zurich; 19.11. Hôtel Central Plaza, Zurich. 